\title{
PREGNANCY LOSS AND DISEASE ACTIVITY SIX MONTHS BEFORE PREGNANCY IN MOTHERS WITH SYSTEMIC LUPUS ERYTHEMATOSUS IN A TERTIARY HOSPITAL
}

Sergio Henrique Oliveira dos Santos ${ }^{1 *}$, Juliana Bühring ${ }^{1}$, Bárbara Seabra Carneiro ${ }^{1}$, Domingos Sávio Nunes de Lima²

1. Hospital Universitário Getúlio Vargas, Manaus (AM), Brazil. 2. Universidade Federal do Amazonas, Manaus (AM), Brazil.

*Corresponding author: sergio.henrique@live.com

\section{BACKGROUND}

Systemic lupus erythematosus (SLE) is an autoimmune disease that has a variable clinical picture and a remitting-recurrent character. It can reach a female:male ratio 13:1, affecting negatively maternal reproductive aspect. Disease activity (DA) six months before pregnancy is a well-known risk factor to pregnancy loss (PL), the reported frequency of $\mathrm{PL}$ is about $16 \%$. The present study aimed to describe the frequency of DA and PL in pregnancies of SLE patients.

\section{MATERIALS AND METHODS}

A descriptive cross-sectional study was performed in a rheumatology service of a tertiary hospital that follows about 800 patients. The patients who had at least one pregnancy from January 2003 until December 2019 and was in regular follow-up were included. Patients unable to provide data about the pregnancy outcome were excluded; categorical variable was correlated using the Fisher's exact test and the proportions were tested using the binomial test.

\section{RESULTS}

One hundred and thirty-one pregnancies were included with 95 patients; the mean age at pregnancy was 28.32 years, standard deviation (SD) 5.6 years. The mean disease length was 6.46 years SD 4.55 years. Sixty-three $(48.1 \%, p=0.727)$ pregnancies was experiencing a flare six months before pregnancy in one or more clinical aspect (27.5\% renal, $19.8 \%$ cutaneous, $6.1 \%$ joint, $3.1 \%$ hematological) (Figs. 1-3). Thirty-two $(24 \%, p<0.008)$ pregnancies ended in PL and there was significant difference (68.1 vs. $31.2 \%, p=0.025$ ) in the incidence of $P L$ in active disease group. There was also positive association between activity six month before pregnancy and any kind of activity and $\mathrm{PL}(\mathrm{p}=0.007$, odds ratio $\mathrm{OR}=3.11,95 \% \mathrm{Cl}=1.33-7.26)$, renal activity (RA) and $\mathrm{PL}(\mathrm{p}=0.018, \mathrm{OR}=2.72,95 \% \mathrm{Cl}=1.17-6.33)$. There was no significant association between others types of $\mathrm{DA}$ and $\mathrm{PL}$. The presence of double stranded DNA antibody (anti-ds-DNA) in any time was also associated with $\mathrm{PL}(\mathrm{p}=0.011, \mathrm{OR}=2.82,95 \% \mathrm{Cl}=1.22-6.47$ ).

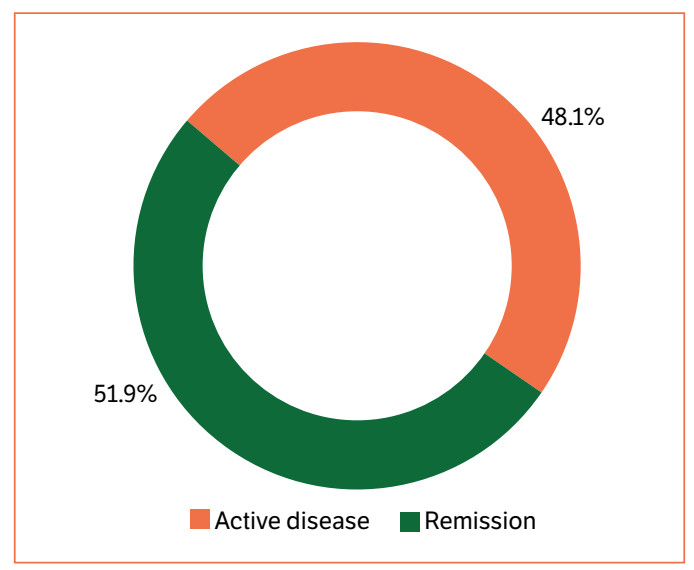

Figure 1. Distribution of frequency of disease activity six months before pregnancy. 


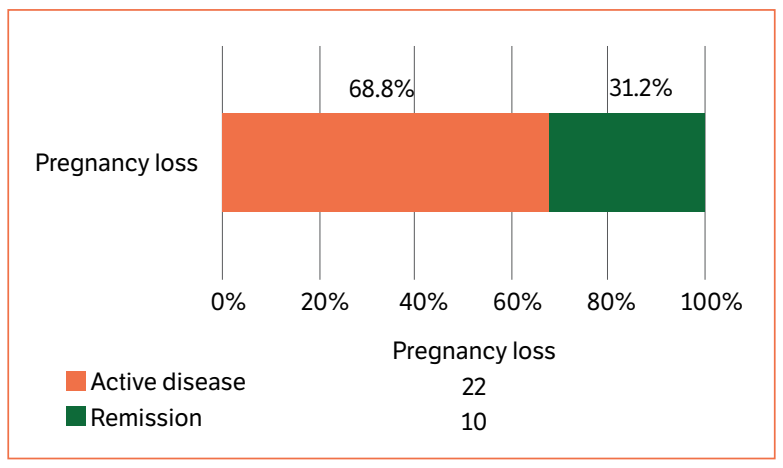

Figure 2. Frequency of pregnancy loss by disease activity six months before pregnancy.

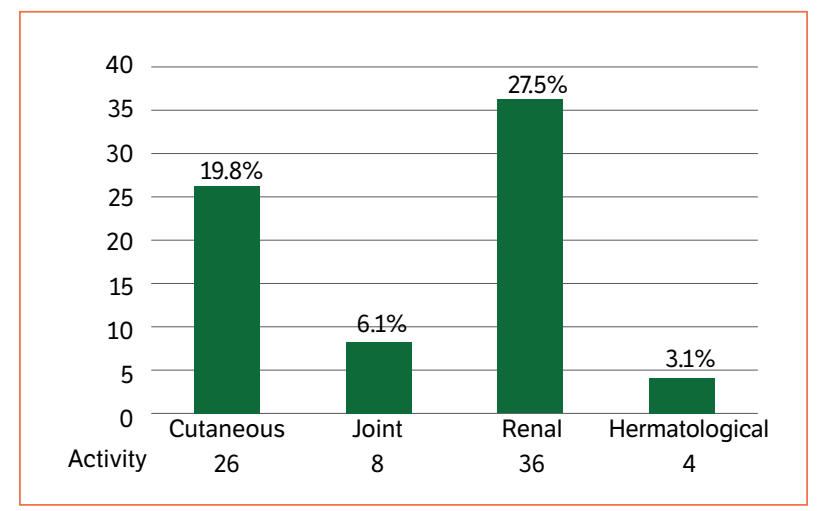

Figure 3. Frequency of specific clinical activity six months before pregnancy.

\section{CONCLUSION}

In our sample, we could verify that a considerable proportion of pregnancies ended in PL and demonstrate that disease activity six month before pregnancy was associated with PL. Although we could not note significant association between all kinds of clinical activity and PL, we could verify a dependent relationship between renal activity and PL as well as the presence of anti-ds-DNA in any time and PL. Our findings are in concert with current literature, except to frequency of PL that was significantly higher than reported. 